\title{
DIFFERENTIATION IN BANACH SPACES
}

APPROVED :

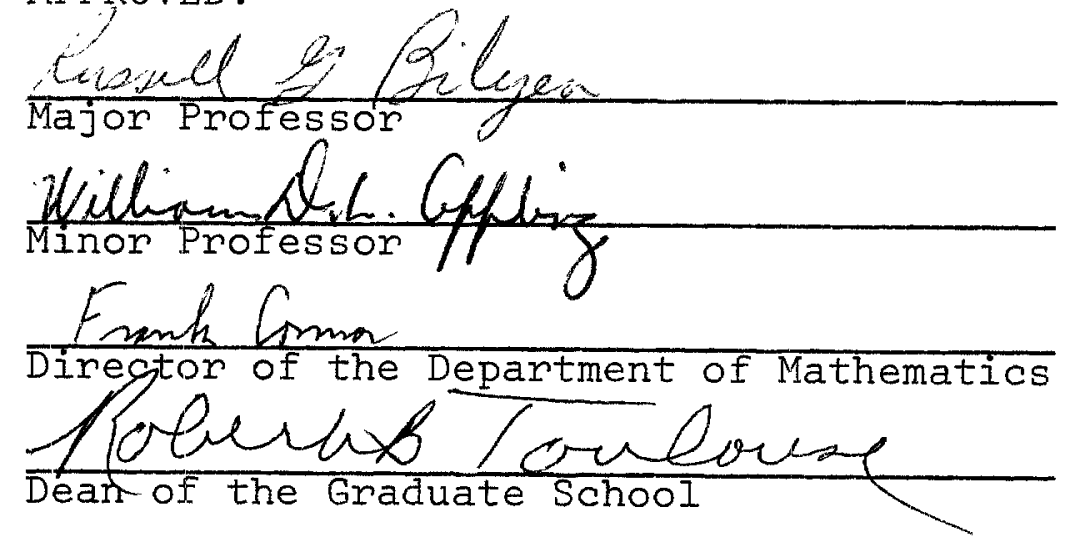


Heath, James Darrell, Differentiation in Banach Spaces. Master of Science (Mathematics), December, 1972, 27 pp., Bibliography, 3 titles.

This thesis investigates the properties and applications of derivatives of functions whose domain and range are Banach spaces. The material is presented in two chapters. In Chapter I, the definition of the derivative is given, and its basic properties are discussed. In Chapter II, stronger conditions are imposed on the derivative, and certain applications--such as proving, under suitable hypotheses, the existence of local inverse and implicit functions and of generalized Lagrange multipliers--are presented. 


\title{
DIFFERENTIATION IN BANACH SPACES
}

\section{THESIS}

\author{
Presented to the Graduate Council of the \\ North Texas State University in Partial \\ Fulfillment of the Requirements
}

For the Degree of

\begin{abstract}
MASTER OF SCIENCE
\end{abstract}
By

\author{
James Darrell Heath, B.S. \\ Denton, Texas \\ December, 1972
}




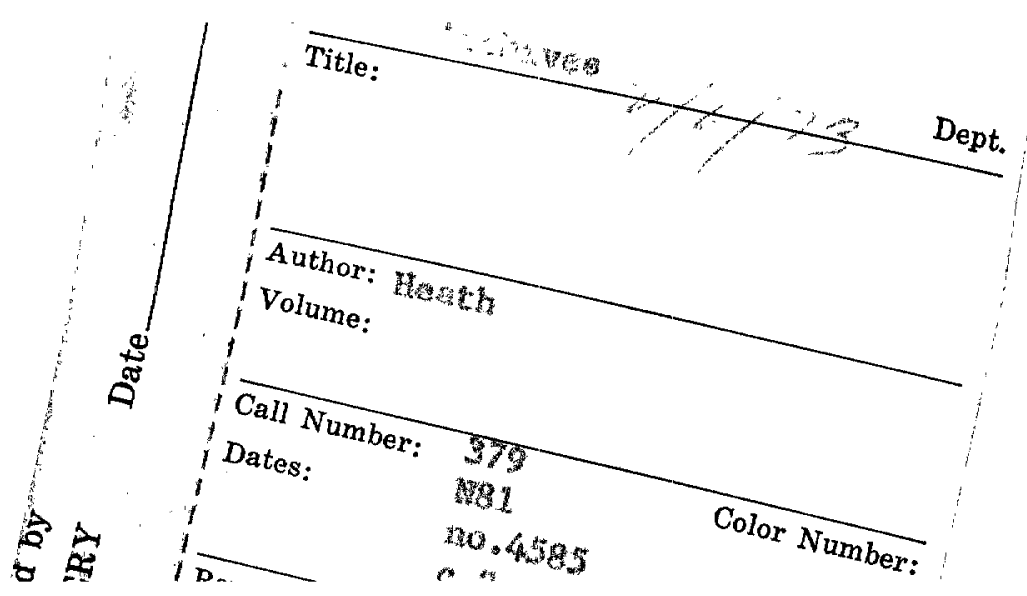


TABLE OE CONTENTS

Chapter
I. INTRODUCTION. . . . . . . . . . . . . . 1
II. APPLICATIONS. . . . . . . . . . . . . . . 1 
CEAPTER I

\section{INTRODUCTION}

The purpose of this study is to define and study the properties of a derivative which is applicable for functions whose domain and range are Banach spaces. Chapter I is primarily concerned with the basic definitions and properties of these derivatives, and Chapter II is mainly devoted to applications of the basic theory.

It is assumed that the reader has an elementary knowledge of limits, inequalities, and Banach spaces. He should also be aware of the basic properties of linear transformations.

Throughout this paper, unless otherwise specified, E, $F$, and $G$ will denote Banach spaces, and $A$ and $B$ will denote non-empty, open subsets of $E$ and $F$ respectively. The functions $f$ and $f^{\prime}$ will denote mappings from $A$ into $F$, and $g$ will be a function from $B$ into $G$. $R$ will denote the set of real numbers.

Definition 1.I: The function $f$ has a directional derivative at $\mathrm{x} \in \mathrm{A}$ in the direction, $\mathrm{y} \in \mathrm{E}$, written. $D_{f(x)}(y)$, if for each $\varepsilon>0$, there exists $\delta>0$ such that if $|t|<\delta,\left\|f(x+t y)-f(x)-D_{f(x)}(t y)\right\| \leq \varepsilon|t|$. The function $f$ is said to be differentiable at $x$ if $D_{f(x)}(y)$ 
exists for all $y \in E$. Since $A$ is an open set, if $x \in A$ and $y \in E$, then $x+t y \in A$ for all sufficiently small t's, $t \neq 0$; it will be tacitly assuned that the $t$ 's chosen throughout this study satisfy this requirement.

Theorem 1.I: If $D_{f(x)}(y)$ and $D_{f^{\prime}(x)}(y)$ exist, then $D_{\left(f+f^{\prime}\right)(x)(y)=D_{f(x)}(y)+D_{f}(x)}(y)$.

Proof: Let $\varepsilon>0$. There exists $\delta_{1}>0$ such that if $|t|<\delta_{1}$, then $\left\|f(x+t y)-f(x)-t D_{f(x)}(y)\right\| \leq|t| \varepsilon / 2$; there exists $\delta_{2}>0$ such that if $|t|<\delta_{2}$, then $\left\|f^{\prime}(x+t y)-f^{\prime}(x)-t D_{f^{\prime}(x)}(y)\right\| \leq|t| \varepsilon / 2$. Let $\delta=\min \left\{\delta_{1}, \delta_{2}\right\}$ so if $|t|<\delta$, then $\| f(x+t y)-f(x)-$ $t D_{f(x)}(y)+f^{\prime}(x+t y)-f^{\prime}(x)-t D_{f^{\prime}(x)}(y) \| \leq$ $|t| \varepsilon / 2+|t| \varepsilon / 2$ and $\left\|\left(f+f^{\prime}\right)(x+t y)-\left(f+f^{\prime}\right)(x)-t\left(D_{f(x)}(y)+D_{f^{\prime}(x)}(y)\right)\right\| \leq$ $|t| \varepsilon$.

Theorem 1.2: If $D_{f(x)}(y)$ exists and $k \in R$, then $D_{(k f)(x)}(y)=k D_{f(x)}(y)$.

Proof: Let $\varepsilon>0$. If $k=0$ and $t \in R$, then $\left\|(0 \cdot f)(x+t y)-(0 \cdot f) x-t\left(0 \cdot D_{f(x)}(y)\right)\right\|=0 \leq|t| \varepsilon$. If $k \neq 0$, there exists $\delta>0$ such that if $|t|<\delta$, then $\left\|f(x+t y)-f(x)-t D_{f(x)}(y)\right\| \leq|t| \varepsilon /|k|$, and on multiplying by $|k|$ the above becomes $\left\|k f(x+t y)-k f(x)-t\left(k D_{f(x)}(y)\right)\right\| \leq|t| \varepsilon$. Theorem 1.3: If $D_{f(x)}(y)$ exists and $k \in R$, then $D_{f(x)}(k y)=k D_{f(x)}(y)$. 
Proof: Let $\varepsilon>0$. If $k=0$ and $t \in R$, $\left\|f(x+t(0 y))-f(x)-t\left(0 \cdot D_{f(x)}(y)\right)\right\|=0 \leq \varepsilon|t|$. If $k \neq 0$, there exists $\delta>0$ such that if $|t|<\delta$, $\left\|f(x+t k y)-f(x)-t k D_{f(x)}(y)\right\| \leq(\varepsilon /|k|)|t k|=\varepsilon|t|$.

Theorem 1.4: Let $a, b \in A$ and $f$ be continuous on the closed line segment from $a$ to $b$, and let $D_{f(x)}(b-a)$ exist for each $y$ on the open line segment from $a$ to $b$; then there exists $z \in L=\{y: y=a+k(b-a), 0<k<l\}$ such that $D_{f(z)}(b-a)=f(b)-f(a)$.

Proof: Let $h=b-a$, and let $g: R \rightarrow R$ where $g(k\|h\|)=f(a+k h), k \in[0, I]$. Then g satisfies the hypothesis for the mean value theorem for functions from $R$ to $R$. Therefore there exists $k \in(O, I)$ such that $g^{\prime}(k\|h\|)=[g(\|h\|)-g(0)] /\|h\|$. Now $D_{f(a+k h)}(h)=\lim _{t \rightarrow 0}[f(a+k h+t h)-f(a+k h)] / t=$ $\left.\lim _{t \rightarrow 0}[g[k+t)\|h\|]-g(k\|h\|)\right] / t=$ $\|h\| g^{\prime}(k\|h\|)=\|h\|\{[g(\|h\|)-g(0)] /\|h\|\}=f(b)-f(a)$. It will now be shown that requiring certain conditions on $f$ and $D_{f}$ will result in $D_{f}$ being linear. By Theorem 1.3, $D_{f}$ is homogeneous so it must be shown that under special conditions $D_{f}$ is additive. For the proofs to follow, the following equivalent definition of $D_{f(x)}(y)$ will be used: $D_{f(x)}(y)=\lim _{t \rightarrow 0}[f(x+t y)-f(x)] / t$. 
Theorem 1.5: Suppose $f, D_{f}$ satisfy the following:

(I) For every $\varepsilon>0$, there exists $\delta>0$ such that if

$x_{0}, x \in A, y \in E,\|y\| \leq I,\left\|x-x_{0}\right\|<\delta$, then

$\left\|D_{f(x)}(y)-D_{f\left(x_{0}\right)}(y)\right\|<\varepsilon$.

(2) For every $\varepsilon>0$, there exists $\delta>0$ such that

$$
\left\|D_{f(x)}(y)-[f(x+t y)-f(x)] / t\right\|<\varepsilon \text { for }|t|<\delta .
$$

Then if $y_{1}, z_{1} \in E, D_{f(x)}\left(y_{1}+z_{1}\right)=$

$D_{f(x)}\left(y_{1}\right)+D_{f(x)}\left(z_{1}\right)$.

Proof: The theorem is obvious if $y_{1}$ or $z_{3}$ equals zero; therefore, assume both are non-zero. Let $\alpha=\max \left\{\left\|y_{1}\right\|,\left\|z_{1}\right\|,\left\|y_{1}+z_{1}\right\|\right\}$. Then $D_{f(x)}\left(y_{1}+z_{1}\right)=\alpha D_{f(x)}(y+z)$ where $y=y_{1} / \alpha, z=z_{1} / \alpha$, and by the choice of $\alpha,\|y\|,\|z\|,\|y+z\| \leq 1$. Let $\varepsilon^{\prime}>0$, and let $\varepsilon=\varepsilon^{\prime} / \alpha$; then there exists $\delta_{2}>0$ such that if $|t|<\delta_{2}$ and $\left|t_{0}\right|<\delta_{1}$, then $\left\|D_{f\left(x+t_{0}\right)}(z)-\left[f\left(x+t_{0} y+t z\right)-f\left(x+t_{0} y\right)\right] / t\right\|<\varepsilon / 8$. Also there exists $\delta_{3}$ such that if $|t|<\delta_{3}$ and $t_{0}<\delta_{1}$, then $\left\|D_{f\left(x+t_{0} z\right)}(y)-\left[f\left(x+t_{0} z+t y\right)-f\left(x+t_{0} z\right)\right] / t\right\|<\varepsilon / 8$. By assumption ( 1 ) of the hypothesis, there exists $\delta_{4}$ such that if $\left\|x-\left(x+t_{0} y\right)\right\|<\delta_{4}$, then $\left\|D_{f(x)}(z)-D_{f\left(x+t_{0 y}\right)}(z)\right\|<\varepsilon / 8$; and there exists $\delta_{5}$ such that if $\left\|x-\left(x+t_{0} z\right)\right\|<\delta_{5}$, then $\left\|D_{f(x)}(y)-D_{f\left(x+t_{0} z\right)}(y)\right\|<\varepsilon / 8$. Let $\delta=\min \left\{\delta_{1}, \cdots, \delta_{5}\right\}$, and let $|t|<\delta$ and $t_{0}=t$. 
Then, $\left\|[f(x+t z+t y)-f(x+t z)] / t-D_{f(x+t z)}(y)\right\|+$ $\left\|D_{f(x+t z)}(y)-D_{f(x)}(y)\right\|<\varepsilon / 8+\varepsilon / 8$ which implies $\left\|[f(x+t z+t y)-f(x+t z)] / t-D_{f(x)}(y)\right\|<\varepsilon / 4$. Adding $\left\|D_{f(x)}(y)-[f(x+t y)-f(x)] / t\right\|<\varepsilon / 8$ to the above, the following is obtained: $\|[-f(x+t z+t y)-f(x)+f(x+t z)+f(x+t y)] / t\|<3 \varepsilon / 8$. This gives $\|\{[f(x+t z+t y)-f(x)]-[f(x+t y+t z)-f(x+t z)]-$ $[f(x+t y+t z)-f(x+t y)]\} \div t \|<3 \varepsilon / 8$. Adding $\left\|D_{f(x)}(y+z)-[f(x+t(y+z))-f(x)] / t\right\|<\varepsilon / 8$, $\left\|[f(x+t z+t y)-f(x+t z)] / t-D_{f(x+t z)}(y)\right\|<\varepsilon / 8$, and $\left\|[f(x+t y+t z)-f(x+t y)] / t-D_{f(x+t y)}(z)\right\|<\varepsilon / 8$ to the previous inequality, the following result is obtained: $\left\|D_{f(x)}(y+z)-D_{f(x+t z)}(y)-D_{f(x+t y)}(z)\right\|<\varepsilon / 6$.

Then recalling $\left.\| D_{f(x}+t z\right)(y)-D_{f(x)}(y) \|<\varepsilon / 8$ and $\left\|D_{f(x+t y)}(z)-D_{f(x)}(z)\right\|<\varepsilon / 8$ and adding these to (i), it follows that

$$
\left\|D_{f(x)}(y+z)-D_{f(x)}(y)-D_{f(x)}(z)\right\|<8 \varepsilon / 8=\varepsilon=\varepsilon^{\prime} / \alpha .
$$

On multiplying the above by $\alpha$,

$$
\left\|D_{f(x)}\left(y_{1}+z_{1}\right)-D_{f(x)}\left(y_{1}\right)-D_{f(x)}\left(z_{1}\right)\right\|<\varepsilon^{\prime} \text {. }
$$

Theorem 1.6: If for $0 \leq a \leq 1$, $f(a x+(1-a) y) \leq a f(x)+(I-a) f(y)$, and if $f$ is differentiable at $x$, then $D_{f(x)}(y+z)=D_{f(x)}(y)+D_{f(x)}(z)$. 
Proof: Let $y^{\prime}=2 y, z^{2}=2 z$. For $t>0$,

$\{f[x+t(y+z)] \cdots f(x)\} / t=$

$\left\{f\left[\left(\frac{x}{2}+\frac{1}{2} t y^{\prime}\right)+\left(\frac{x}{2}+\frac{y}{2} t z^{\prime}\right)\right]-\frac{i(x)}{2}-\frac{f(x)}{2}\right\} / t \leq$

$\frac{7}{2}\left[\left\{f\left(x+t y^{\prime}\right)-f(x)\right\} / t\right]+\frac{1}{2}\left[\left\{f\left(x+t z^{\prime}\right)-f(x)\right\} / t\right]$.

on taking $\lim _{t \rightarrow 0+}$ on both. sides of the above inequality, it is found that $D_{f(x)}(y+z) \leq \frac{1}{2}\left[D_{f(x)}\left(y^{i}\right)+D_{f(x)}\left(z^{\prime}\right)\right]=$ $D_{f(x)}(y)+D_{f(x)}(z)$.

For $t<0$,

$\|\{f[x+t(y+z)]\} / t\| \geq \frac{1}{2}\left\|\left\{f\left(x+t y^{\prime}\right)-f(x)\right\} / t\right\|+$

$\frac{1}{2}\left\|\left\{f\left(x+t z^{\prime}\right)-f(x)\right\} / t\right\|$ and by the previous argument

taking $\lim _{t \rightarrow 0^{-}}$, then $D_{f(x)}(y+z) \geq D_{f(x)}(y)+D_{f(x)}(z)$.

From $(i)$ and $(i i), D_{f(x)}(y+z)=D_{f(x)}(y)+D_{f(x)}(z)$. 


\section{APPLICATIONS}

In this chapter certain results will be shown to exist when stronger conditions are imposed on $D_{f}$.

Definition 2.1: The function $f$ is strongly differentiable at $x \in A$ if there exists a linear transformation, $d_{f(x)}$, from $A$ to $F$ such that for every $\varepsilon>0$ there exists $\delta>0$ such that if $\|t y\|<\delta$, then

$$
\left\|[f(x+t y)-f(x)] / t-d_{f(x)}(y)\right\| \leq \varepsilon\|y\| \text {. }
$$

Theorem 2.1: If $f$ and $f$ are strongly differentiable at $a \in A$ and $k \in R$, then $d_{\left(f+f^{\prime}\right)(a)}=d_{f(a)}+d_{f^{\prime}(a)}$, and $d_{(k f)(a)}=k d_{f(a)}$.

Proof: The proof of this theorem is identical to the proof of Theorem I.I and is omitted.

Theorem 2.2: If $f$ is strongly differentiable at $x \in A$ and $y \in E$, then $D_{f(x)}(y)$ exists.

Proof: This is trivial if $\mathrm{y}=0$, so let $\mathrm{y} \in \mathrm{E}$ and $\varepsilon>0$, and let $\bar{\varepsilon}=e /\|y\|$. Since $f$ is strongly differentiable at $x$, there exists $\delta$ such that if $\|t y\|<\delta$, then $\left\|[f(x+t y)-f(x)] / t-a_{f(x)}(y)\right\| \leq \bar{\varepsilon}\|y\|=\varepsilon$. 
Lemma 2, I: If $I$ is a linear transfomation from $E$ into $F$ and $T$ is continuous at $x_{0} \in E$, then $T$ is continuous on $E$.

Proof: Let $\varepsilon>0$. Since $T$ is continuous at $x_{0}$, there exists $\delta>0$ such that $\left\|T\left(x_{0}\right)-T(x)\right\|<\varepsilon$ when $\left\|x_{0}-x\right\|<\delta$. Let $x_{1} \in E$. Then for all $y \in E$ with
$\left\|x_{1}-y\right\|<\delta,\left\|x_{0}-\left(y-x_{1}-x_{0}\right)\right\|=\left\|x_{1}-y\right\|<\delta$,
and $\left\|T\left(x_{1}\right)-T(y)\right\|=\left\|T\left(x_{0}\right)-T\left(y-x_{1}+x_{0}\right)\right\|<\varepsilon$.

Definition 2.2: A linear transformation $T$ from a Banach space $B_{1}$ into a Banach space $B_{2}$ is bounded if $\{\|\mathrm{T}(\mathrm{x})\|:\|x\| \leq I\}$ is a bounded set of real numbers. If $T$ is bounded, the non-negative real number $\|T\|$ is defined to be the I.u.b. of the set $\{\|\mathrm{T}(\mathrm{x})\|:\|\mathrm{x}\| \leq 1\}$. It is a consequence of this definition that if $x \in B_{1}$, then $\|T(x)\| \leq\|T\|\|x\|$.

Lemma 2.2: If $T$ is a linear transformation from $E$ into $F$, then $T$ is continuous if and only if it is bounded.

Proof: Suppose first that $T$ is continuous on $E$, then $T$ is continuous at 0 . Therefore, since $T(0)=0$, there exists $\delta>0$ such that $\|T(x)\| \leq I$ if $\|x\|<\delta$. Let $\|x\| \leq 1$. Then $\left\|\frac{1}{2} \delta x\right\|=\frac{1}{2} \delta\|x\|<\delta$, and, therefore, $\left\|T\left(\frac{1}{2} \delta x\right)\right\|<1$. Therefore, $\|T(x)\|=\left\|\frac{2}{\delta} T\left(\frac{1}{2} \delta x\right)\right\|=$ $\frac{2}{\delta}\left\|T\left(\frac{1}{2} \delta x\right)\right\|<\frac{2}{\delta}$.

Suppose next that $T$ is bounded. By Lemma 2.1 it will be sufficient to prove that $T$ is continuous at 0 . Let $\varepsilon>0$, and let $M \rightarrow\left\|T_{x}\right\| \leq M$ whenever $\|x\| \leq 1$. 
Let $\delta>0$ such that $\delta M<\varepsilon$. If $\|x\|<\delta$, then $\delta^{n-1}\|x\|<1$, and, therefore, $\left\|T\left(\delta^{-1} x\right)\right\| \leq M$. So for $\|x\|<\delta,\|T(x)\|=\left\|\delta T\left(\delta^{-1} x\right)\right\| \leq \delta M=\varepsilon$.

Theorem 2.3: If $f$ is strongly differentiable at a $E A$, then $d_{f(a)}$ is bounded if and only if $f$ is continuous at $a$. Proof: Suppose first that $f$ is continuous at as and let $\varepsilon>0$. Then there exists $\delta_{1}>0$ and $\delta_{2}>0$ such that $\|f(a+t y)-f(a)\|<\frac{1}{2} \varepsilon$ for $0<\|t y\|<\delta_{1}$, and $\left\|f(a+t y)-f(a)-d_{f(a)}(t y)\right\| \leq \frac{1}{2} \varepsilon\|t y\|$ for $0<\|$ ty $\|<\delta_{2}$. Let $\delta=\min \left\{\delta_{1}, \delta_{2}, I\right\}$ and $\|$ ty $\|<\delta$. Then $\left\|d_{f(a)}(t y)\right\| \leq\left\|d_{f(a)}(t y)-f(a+t y)-f(a)\right\|+$ $\|f(a+t y)-f(a)\|<\frac{1}{2} \varepsilon\|t y\|+\frac{1}{2} \varepsilon<\varepsilon$.

This proves $d_{f(a)}$ is continuous at 0 , and by Lemma 2.1 . and Lemma $2.2, \mathrm{~d}_{f(a)}$ is bounded.

Next suppose $d_{f(a)}$ is bounded. Then there exists $\delta>0$ such that $\left\|f(a+t y)-f(a)-a_{f(a)}(t y)\right\|<1\|t y\|$ for $\|t y\|<\delta$. Let $\|$ ty $\|<\delta$, then $\|f(a+t y)-f(a)\| \leq\left\|f(x)-f(a)-d_{f(a)}(t y)\right\|+$ $\| d_{f(a)}\left(t y\|\leq\| t y\|+\| d_{f(a)}\|\| t y\left\|=\left(1+\left\|d_{f(a)}\right\|\right)\right\| t y \|\right.$. Theorem 2.4: If $f: A \rightarrow B$ and $D_{f(a)}(y)$ exists for some $y \quad B$, and if $g$ is continuous and strongly differentiable at $b=f(a)$, then $\left.D_{[g} f\right](a)(y)=d_{g(b)}\left(D_{f(a)}(y)\right)$.

Proof: Let $0<E_{1}<I$; then there exists $\delta_{1}>0$ such that if $|t|<\delta_{1},\|f(a+t y)-f(a)-t D(y)\| \leq \varepsilon_{1}|t|$ and there exists $\delta_{2}>0$ such that $\left\|g(b+t k)-g(b)-d_{g}(t k)\right\| \leq \varepsilon_{1}\|t k\|$ for $\|t k\|<\delta_{2}$. 
If $|t|<\delta_{1}$, then $\|f(a+t y)-b\| \leq$ $\left\|F(a+t y)-f(a)-D_{f}(t y)\right\|+\left\|D_{f}(t y)\right\| \leq$ $\varepsilon_{1}|t|+|t|\left\|D_{f(a)}(y)\right\|<\left(I+\left\|D_{f(a)}(y)\right\|\right)|t|$. Let $\delta=\min \left\{\delta_{1}, \delta_{2}\left(1+\left\|D_{f(a)}(y)\right\|\right)^{-1}\right\}$, and let $|t|<\delta$. Then $\|f(a+t y)-b\|<\delta_{2}$, and, therefore, $\left\|g(f(a+t y))-g(f(a))-d_{g(b)}\left(D_{f(a)}(t y)\right)\right\| \leq$ $\left\|g(f(a+t y))-g(b)-d_{g(b)}(f(a+t y)-b)\right\|+$ $\left\|d_{g(b)}(f(a+t y)-b)-d_{g(b)} D_{f(a)}(t y)\right\| \leq$ $\varepsilon_{1}\|f(a+t y)-b\|+\left\|d_{g(b)}(f(a+t y)-b)-a_{g(b)} D_{f(a)}(t y)\right\|$ $\leq \varepsilon_{1}|t|\left(I+\left\|D_{f(a)}(y)\right\|\right)+\left\|d_{g(b)}\right\| \| f(a+t y)-b-$ $D_{f(a)}(t y) \| \leq \varepsilon_{1}\left(1+\left\|D_{f(a)}(y)\right\|\right)|t|+d_{g(b)}\left|\varepsilon_{1} t\right| \leq$ $\varepsilon_{1}|t|\left(1+\left\|D_{f(a)}(y)\right\|+\left\|d_{g(b)}\right\|\right)$. Let $\varepsilon>0$ and $\bar{E}=\min \left\{1, \varepsilon\left(1+\left\|D_{f(a)}(y)\right\|+\left\|d_{g(b)}\right\|\right)^{-1}\right\}$. Then by what has just been shown, there exists $\delta>0$ such that jf $0<|t|<\delta$, then $\left\|g(f(a+t y))-g(f(a))-d_{g(b)}\left(D_{f(a)}(t y)\right)\right\|<\varepsilon|t|$. Theorem 2.5: Let $a, b \in A$, and suppose that $L=$ $\{a+k(b-a): 0 \leq k \leq 1\}$ is contained in $A$. Suppose further that there exists $M \in R$ such that $\left\|d_{f(x)}\right\| \leq M$ for all $x \in L$. If $f$ is continuous on $L$, then $\|f(b)-f(a)\| \leq M\|b-a\|$.

Proof: Let $c=b-a$ and $g(k)=f(a+k c)$ for $0 \leq k \leq 1$. For each $\varepsilon>0$, let $J_{\varepsilon}=\{k \in[0,1]: \quad\|g(n)-g(0)\| \leq(M+\varepsilon) n\|c\|$ for $0 \leq n \leq k\}$. Clearly, $0 \in J_{\varepsilon}$. Let $\alpha=$ l.u.b. $J_{\varepsilon}$. It will be shown that $\alpha \in J_{\varepsilon}$. This is obviousiy true if $\alpha=0$; 
therefore, assume $\alpha>0$. For each $\mathrm{n}$ with $0 \leq n<\alpha$ there exists $k_{n} \in J_{E}$ with $n<k_{n}$, and by jefinition of $J_{E}$, $\|g(n)-g(0)\| \leq(M+\varepsilon)_{n}\|c\|$.

Since $f$ is continuous on $L$, then $g$ is continuous on $[0,2]$.

Then by the above and the continuity of $\mathrm{g}$, $\|g(\alpha)-g(0)\|=\lim _{\delta \rightarrow 0}\|g(\alpha-\delta)-g(0)\| \leq$

$\lim _{\delta \rightarrow 0}(M+\varepsilon)(\alpha-\delta)\|c\|=(M+\varepsilon)(\alpha)\|c\|$.

Therefore $\alpha \in J_{\varepsilon}$.

It will now be shown that $\alpha=1$. Suppose $\alpha<1$.

Let $x=a+a c$. Then $f$ is differentiable at $x$, so there exists $\delta_{1}>0$ such that

$\left\|f(x+t y)-f(x)-t D_{f(x)}(y)\right\| \leq \varepsilon\|t y\|$ for $\|t y\|<\delta_{1}$. Since $x \quad L,\left\|a_{f(x)}\right\| \leq M$; therefore, for $\|t y\|<\delta_{1}$, the following inequality holds:

$$
\begin{aligned}
& \|f(x+t y)-f(x)\| \leq f(x+t y)-f(x)-t d_{f(x)}(y) \|+ \\
& \left\|t d_{f(x)}(y)\right\| \leq \varepsilon\|t y\|+|t|\left\|a_{f(x)}(y)\right\| \leq \\
& \varepsilon\|t y\|+|t|\|M\|\|y\|=(M+\varepsilon)\|t y\| .
\end{aligned}
$$$$
\text { Let } \sigma \text { exist such that } \alpha<\sigma \leq I \text { and }(\sigma-\alpha)\|c\|<\delta_{1} \text {. }
$$$$
\text { If } \alpha<\mathrm{n} \leq \sigma \text { and } \mathrm{x}+\mathrm{ty}=\mathrm{a}+\mathrm{nc} \text {, then } \| \text { ty }\|=\| \mathrm{x}+\mathrm{ty}-\mathrm{x} \|
$$$$
=\|a+n c-x\|=\|a+n c-a-a c\|=(n-\alpha)\|c\| \leq
$$$$
(\sigma-\alpha)\|c\|<\delta_{1} \text {. Hence, (iii) gives }
$$$$
\|g(n)-g(\alpha)\|=\|f(x+t y)-f(x)\| \leq(M+\varepsilon)\|t y\|=
$$$$
(M+\varepsilon)(n-\alpha)\|c\| \text {. }
$$

Then, from (ii) and (iv) if $\alpha<n \leq \sigma$,

$\|g(n)-g(0)\| \leq\|g(n)-g(\alpha)\|+\|g(\alpha)-g(0)\| \leq$

$(M+\varepsilon)(n-\alpha)\|c\|+(M+\varepsilon)(\alpha)\|c\|=(M+\varepsilon)(n)\|c\|$. (v) 
Then from (i), ( $i i)$, and ( $v$ ), $\sigma \in J_{\varepsilon}$, which contradicts the assumption that $I>\alpha=I \cdot$ u.b. $J_{\varepsilon}$. Therefore, $\alpha=I$, and $\alpha \in J_{\varepsilon} \cdot$ By definition of $J_{\varepsilon}$, $\|f(b)-f(a)\|=\|g(1)-g(0)\| \leq(M+\varepsilon)\|c\|=$ $(M+\varepsilon)\|b-a\|$. Since $\varepsilon$ is arbitrary, $\|f(b)-f(a)\| \leq M\|b-a\|$.

Lemma 2.3: If $T$ is a linear transformation from $E$ into $F$, then $T$ is strongly differentiable on $E$, and $d_{T(a)}=T$ for all $a \in E$. Consequently, if $T$ is a bounded linear transformation, then $T$ is continuousiy differentiable on $E$.

Proof: $\|T(x+t y)-T(x)-T(t y)\|=0$ for $a l l$ $x, y \in E$.

Theorem 2.6: Let $\mathrm{a}$ and $\mathrm{b}$ be distinct points of $\mathrm{A}$, and suppose that $\sigma=\{a+e(b-a) ; 0 \leq e \leq 1\}$ is contained in A. Let $T$ be a bounded linear transformation from $E$ into $F$, and suppose that there exists $M \in R+\left\|d_{f(x)}-T\right\| \leq M$ for all $x \in \sigma$. Then $\|f(b)-f(a)-T(b-a)\| \leq M\|b-a\|$.

Proof: Let $h(x)=f(x)-T(x)$. From Theorem $2 \cdot 1$ and Lemma $2.3, h$ is differentiable on $\sigma$ and $d_{h(x)}=d_{f(x)}-T$ for all $x \in \sigma$. Therefore, $\left\|d_{h(x)}\right\| \leq M$ for all $x \in \sigma$, and Theorem 2.5 gives $\|f(b)-f(a)-T(b-a)\|=\|h(b)-h(a)\| \leq M\|b-a\|$. Definition 2.3: Let $B_{1}$ be a Banach space and $x \in B_{1}$ and $r \in R$, then $B(x, r)=\left\{y \in B_{1}:\|x-y\|<r\right\}$ and $\bar{B}(x, r)=\left\{y \in B_{1}: \quad\|x-y\| \leq r\right\}$. 
Theorem 2.7: Let $B=B(z, r)$ for $z \in E$ and $n \in R$, and let $T$ be a bounded linear trensformation from $E$ into $F$. Suppose there exists $M \in \mathbb{R}$ such that

$$
\begin{aligned}
& \left\|d_{f(x)}-T\right\| \leq M \text { for } a l l x \in B \text {. Then } \\
& \|f(b)-f(a)-T(b-a)\| \leq M\|b-a\| \text { for } a l l a, b \in B .
\end{aligned}
$$

Proof: The line segment $\sigma=\{a+e(b-a): 0 \leq e \leq 1\}$ is contained in $B$ for all $a, b \in B$; apply Theorem 2.6.

Definition 2.4: Let $S_{\sigma}$ be any subset of $E$, and Jet $x_{0}$ be any accumulation point of $S_{0}$, and let $S=S_{0} \backslash\left\{x_{0}\right\}$. Then the ray from $x_{0}$ through $x$ is said to tend to a limiting position as $x \rightarrow x_{0}, x \in S$, if $\underset{x \rightarrow x_{0}}{\lim _{x \rightarrow 0}} \frac{x-x_{0}}{\| x-x_{0}}$ exists. The value of this limit is obviously a unit vector $e \in E$, and a ray with origin $x_{0}$ in the direction $e$ is said to be a tangent ray to $S_{0}$ at $x_{0}$.

Given a set $A \subset E$ and an accumulation point $x_{0}$ of $A$, then consider all subsets $A_{0}$ of $A$ possessing a tangent ray at $x_{0}$; the union of all the tangent rays so obtained is the tangent cone to $A$ at $x_{0}$.

Theorem 2.8: Let $S$ be an open subset of $E$, and let $f: s \rightarrow F$. Suppose $d_{f(x)}$ is linear and continuous at $x=0$, $f(0)=0$, and range $d_{f(0)}=F$. Let $q$ be a unit vector in $E$, and let $L=\left\{\lambda d_{f(0)}(q): \lambda \in R, \lambda \geq 0\right\}$. Then there exists $\left\{x_{n}\right\}_{n=1}^{\infty}$ of non-zero points of $f^{-1}(L)$ such that $\lim _{n \rightarrow \infty} x_{n}=0$ and $\lim _{n \rightarrow \infty} x_{n} /\left\|x_{n}\right\| \rightarrow q$. 
Proof: To prove this theorem, the following two lemuas are needed. The first is a consequence of the open mapping theorem and is stated without proof.

Lemma 2.4: Let $u$ be a continuous linear function from $E$ onto $F$. Then there exists a positive number $k$ such that for all $y \in F$, there exists $x \in E$ such that $u(x)=y$ arid $\|\mathrm{x}\| \leq \mathrm{k}\|\mathrm{y}\|$.

Lemma 2.5: Let $k, \varepsilon$ be real numbers with $k>0$ and $0<\varepsilon<1$, and let $u$ be a continuous linear function from $E$ onto $F$ with the property that for each $y \in F$, there exists $x \in E$ such that $u(x)=y$ and $\|x\| \leq k\|y\|$. Also let $f$ be a continuous function from a subset of $E$ into $F$ such that $0 \in \operatorname{domain}(f), f(0)=0$, and $\left\|f(x)-u(x)-\left[f\left(x^{\prime}\right)-u\left(x^{\prime}\right)\right]\right\| \leq \varepsilon / k\left\|x-x^{\prime}\right\|$

for all $x, x^{\prime} \in$ domain ( $\left.f\right)$. Let $z$ be a point of domain ( $f$ ) such that domain ( $f)$ contains $B\left(z, \frac{\varepsilon\|z\|}{1-\varepsilon}\right)$. Then there exists $x \in B\left(z, \frac{\varepsilon\|z\|}{I-\varepsilon}\right)$, such that $f(x)=u(z)$.

Proof: Define $x_{0}=z$, and for $n \geq 0$ let $x_{n}+1$ be a point of $E$ such that $u\left(x_{n+1}-x_{n}\right)=u(z)-f\left(x_{n}\right)$ and $\left\|x_{n+1}-x_{n}\right\| \leq k\left\|u\left(x_{n+1}-x_{n}\right)\right\|$. Obviously $x_{1}$ is defined and by (i), (ij), and (iii), $\left\|x_{1}-z\right\|=\left\|x_{1}-x_{0}\right\| \leq k\left\|u\left(x_{1}-x_{0}\right)\right\|=k\|u(z)-f(z)\|=$ $k\|[f(z)-u(z)]-[f(0)-u(0)] \leq \varepsilon\| z-0 \| \leq \frac{\varepsilon\|z\|}{1-\varepsilon \cdot \text { (iv) }}$ Therefore, $x_{1} \in B\left(a, \frac{\varepsilon\|z\|}{1-\varepsilon}\right)$. It will now be shown by 
induction that $x_{n} \in B\left(z, \frac{\varepsilon\|z\|}{1-\varepsilon}\right)$ for all n. suppose $x, \ldots, x_{n}$ are defined and belong to $B\left(z, \frac{\varepsilon\|z\|}{1-\varepsilon}\right)$.

Define $x_{n+1}$ as above; then for $p=1, \ldots, n$ $\left\|x_{p+1}-x_{p}\right\| \leq k\left\|u\left(x_{p+I}-x_{p}\right)\right\|=k\left\|u\left(x_{p+I}\right)-u\left(x_{p}\right)\right\|=$ $k \|\left[u(z)+u\left(x_{p}\right)-f\left(x_{p}\right)\right]-\left[u(z)+u\left(x_{p-1}\right)-f\left(x_{p-1}\right) \|=\right.$ $k\left\|\left[f\left(x_{p}\right)-u\left(x_{p}\right)\right]-\left[f\left(x_{p-1}\right)-u\left(x_{p-1}\right)\right]\right\| \leq \varepsilon\left\|x_{p}-x_{p-1}\right\|$. Also for $\mathrm{p}=1, \ldots, \mathrm{n}$ $\left\|x_{p+1}-x_{p}\right\| \leq \varepsilon\left\|x_{p}-x_{p-1}\right\| \leq \varepsilon^{2}\left\|x_{p-1}-x_{p-2}\right\| \leq . \cdot$ $\leq \varepsilon^{\mathrm{P}}\left\|\mathrm{x}_{1}-\mathrm{x}_{0}\right\|$.

Therefore, $\left\|x_{n+1}-x_{0}\right\| \leq\left\|x_{n+1}-x_{\Omega}\right\|+\ldots+\left\|x_{1}-x_{0}\right\| \leq$ $\left(\varepsilon^{n}+\varepsilon^{n-1}+\ldots++1\right)\left\|x_{1}-x_{0}\right\|<\frac{\left\|x_{1}-x_{0}\right\|}{1-\varepsilon} \leq$ $\frac{\varepsilon\|z\|}{1-\varepsilon}$ from (iv). Hence, $x_{\mathrm{n}+I} \in B\left(z, \frac{\varepsilon\|z\|}{I-\varepsilon}\right)$.

Therefore, ( $v$ ) holds for all $p$ so if $m$, $n$ are non-regative integers and $n>m \geq 0$, then $\left\|x_{n}-x_{m}\right\| \leq\left\|x_{n}-x_{n-1}\right\|+\ldots+\left\|x_{m+1}-x_{n n}\right\| \leq$ $\left(\varepsilon^{n-I}+\cdots+\varepsilon^{m}\right)\left\|x_{2}-x_{0}\right\|<\frac{\varepsilon^{m}\left\|x_{1}-x_{0}\right\|}{I-\varepsilon}$ which implies $\left\{x_{n}\right\}_{n=0}^{\infty}$ is a Cauchy sequence so $\left\{x_{n}\right\}_{n=0}^{\infty}$ converges to some $\bar{x} \in B\left(z, \frac{\varepsilon\|z\|}{1-\varepsilon}\right)$ and from the way the $x_{n}$ 's were chosen $f(\bar{x})=u(z)$.

Using the above two lemmas, the proof of the theorem can now be completed. The conditions of Lemma 2.4 are satisfied by $d_{f(0)}$, so there exists $k$ such that if 
$y \in F$, there exists $x \in E$ such that $d_{I(0)}(x)=y$ and $\left\|d_{f(0)}(x)\right\| \leq k\|y\|$. Since $f$, by hypothesis, has a continuous derivative at 0 , and since $d_{f(0)}$ is Iinear, then $d_{f-d_{f(0)}}$ is continuous at 0 , and its differential at 0 is the zero function from $E$ into $F$. Since $d_{f-d_{f(0)}}$ is continuous at 0 , given $\varepsilon$ where $0<\varepsilon<1 / 2$, there exists neighborhood, $N$, of 0 with $N$ contained in domain ( $f$ ) such that if $x \quad N$, then $\left\|d_{\left[f-d_{f(0)}\right.}(x)\right\| \leq \varepsilon / k$. Then from Theorem 2.7, $\left\|\left[f(x)-d_{f(0)}(x)\right]-\left[f\left(x^{\prime}\right)-d_{f(0)}\left(x^{\prime}\right)\right]\right\| \leq$ $\varepsilon / k\left\|x-x^{\prime}\right\|$ for $x, x^{\prime} \in N$. There exists $\lambda_{0}$ such that if $0<\lambda<\lambda_{0}$ then $N$ contains $B\left(\lambda q, \frac{\varepsilon \lambda}{1-\varepsilon}\right)$. Then by Lemma 2.5 , for each sufficiently small $\lambda$ there exists $x \neq 0$ such that $\|x-\lambda q\| \leq \varepsilon\|\lambda q\| / I-\varepsilon$ and $f(x)=d_{f(0)}(\lambda q) \in L$, and, therefore, $x \in f^{-1}(L)$. Now applying this result and letting $\varepsilon$ take on the values $1 / 3,1 / 4, \ldots ., 1 / n, \ldots$. and for $\varepsilon=1 / n$, let $\lambda=\lambda n$ where $\lambda_{n} \rightarrow 0$ as $n \rightarrow \infty$. Thus, a sequence $\left\{x_{n}\right\}$ of non-zero elements of $f^{-1}(L)$ and a sequence $\left\{\lambda_{n}\right\}$ of positive numbers with limit zero are obtained such that $\lim _{n \rightarrow \infty} x_{x_{n}}=0$ and such that. $\left\|x_{n}-\lambda_{n} q\right\| \leq \frac{\varepsilon_{n}\left\|\lambda_{n} q\right\|}{I-\varepsilon_{n}}$; so $\frac{\left\|x_{n}-\lambda_{n q}\right\|}{\|\lambda n q\|} \leq \frac{\varepsilon_{n}}{1-\varepsilon_{n}}$ and since $\varepsilon_{n}+0$, then $\lim _{n \rightarrow \infty} \frac{\left\|x_{n}-\lambda_{n q}\right\|}{\|\lambda n q\|}=0$. For any non-zero $x, z \in E$, $\left\|\frac{x}{\|x\|}-\frac{z}{\|z\|}\right\|=\| \frac{(\|z\|-\|x\|) x}{\|x\| \pi z \|}+\frac{x-z \|}{\|z\|} \mid \leq \frac{2\|x-z\|}{\|z\|}$. Let $x_{n}$ be $x$, and $\lambda q$ be $z$ in the above inequality. Then 
$\lim _{n \rightarrow \infty}\left\|\frac{x_{n}}{\left\|x_{n}\right\|}-\frac{\lambda_{n q}}{\left\|\lambda_{n q}\right\|}\right\|=\lim _{n \rightarrow \infty}\left\|\frac{x_{n}}{\left\|x_{n}\right\|}-q\right\| \leq$ $\lim _{n \rightarrow \infty} \frac{2 \| x_{n}-\lambda_{n} d}{\left\|\lambda_{n} q\right\|}=0$. Therefore, $\frac{x_{n}}{\left\|x_{n}\right\|}+q$ as $n \rightarrow \infty$.

Theorem 2.9: Let A be an open subset of $E$, and let $f: A \rightarrow F$. Also let $d_{f}$ be linear and continuous at $x_{0}=0$, and let $d_{f(0)}$ have Range $F$ and $y_{0}=f(0)=0$. Then if $z$ is any closed subspace of $F$, the tangent cone to $f^{-1}\left(y_{0}+z\right)$ at 0 is $\phi^{-1}\left(y_{0}+z\right)$ where $\phi(x)=y_{0}+d_{f\left(x_{0}\right)}\left(x-x_{0}\right)$ for $x \in E$.

Proof: It will first be shown that if $t_{1} \in \phi^{-1}(\mathrm{Z})$, then $t_{1} \in$ tangent cone to $f^{-1}(Z)$. This is trivial if $t_{1}=0$; therefore, assume $t_{1} \neq 0$. Let $t=t_{1} /\left\|t_{1}\right\|$, so $\|t\|=1$, and since $d_{f(0)}$ is linear and $Z$ is a subspace $d_{f(0)}(t) \in Z$. Now $Z \supset L=\left\{\lambda d_{f(0)}(t): \lambda \in R, \lambda \geq 0\right\}$. By Theorem 2.8, there exists a sequence $\left\{x_{n}\right\}$ of non-zero points of $f^{-1}(L)$, therefore of $f^{-1}(Z)$, such that $x_{n} \rightarrow 0$ and $x_{n} /\left\|x_{n}\right\|+t$ as $n \rightarrow \infty$ so that there is a subset of $f^{-1}(Z)$ with a tangent ray at 0 with direction $t$.

It remains to be shown that the tangent cone to $f^{-1}(Z)$ at 0 is contained in $\phi^{-1}(Z)$. If the tangent cone is 0 , there is nothing to prove, so suppose $A_{0} \subset f^{-1}(Z)$ and Ao has a tangent ray at 0 whose direction is a unit vector $q \in E$ so that $p /\|p\| \rightarrow q$ as $p \rightarrow 0$ in the set $A=A_{0} \backslash\{0\}$. Since $d_{f(0)}$ is Iinear and continuous, $\left[d_{f(0)}(p)\right] /\|p\|=$ $d_{f(0)} p /\|p\| \rightarrow d_{f(0)}(q)$ as $p \rightarrow 0$ in $A$. 


$$
\text { Since } \| \lim _{\|y\| \rightarrow 0} \frac{f(t y+0)-f(0)-t d_{f(0)}(y)}{\|t y\|}=0 \text {, }
$$

then $\lim _{\|t y\| \rightarrow 0} \frac{f(t y)}{t\|y\|}=\lim _{\|t y\| \rightarrow 0} d_{f(0)}\left(\frac{y}{\|y\|}\right)$; and since

$\mathrm{d}_{f(0)}$ is continuous, and also since $\frac{\mathrm{y}}{\|\mathrm{y}\|} \rightarrow \mathrm{q}$ as $\mathrm{y} \rightarrow 0 \mathrm{in}$ A, then $d_{f(0)}\left(\frac{y}{\| y}\right) \rightarrow d_{f(0)}(q)$, and, therefore, $\|t y\|^{m} \rightarrow 0 \frac{f(t y)}{\|t y\|}=d_{f(0)}(q)$. Since $f(t y) \in z$ for all ty in $A$, and since $z$ is closed, then $d_{f(0)}(q) \in Z$ and $q \in \phi^{-1}(z)$.

Theorem 2.10: Let $d_{f\left(x_{0}\right)}$ be continuous at $x \in E$, and let $y_{0}=f\left(x_{0}\right)$. Let $\phi(x)=y_{0}+d_{f\left(x_{0}\right)}\left(x-x_{0}\right)$ for $x \in E$. Also let Range $d_{f\left(x_{0}\right)}=F$. Then for any closed vector subspace $Z$ of $Y$, the tangent cone to $f^{-1}\left(y_{0}+Z\right)$ at $x_{0}$ is $\phi^{-1}\left(y_{0}+z\right)$.

Proof: This follows from Theorem 2.8 applied to the function $b(x)=f\left(x+x_{0}\right)-\left(x_{0}\right)$.

Definition 2.5: Let $S=\{x \in E: f(x)=0\}$. If $F: A \rightarrow R$, the $F$ is said to have a non-strict maximum at $x_{0} \in S$ if there exists a neighborhood $N$ of $x_{0}, N \subset A$ such that $F(x) \leq F\left(x_{0}\right)$ for all $x \in S \cap N$. A non-strict minimum is defined similarly.

Theorem 2.11: Let $f: A+G$, and for each $x \in A$ let Range $d_{f(x)}=G$. Let $F: A \rightarrow R$ which is differentiable and continuous on $A$. If $F$ has a non-strict maximum or minimum at $x_{0}$ subject to the condition $f\left(x_{0}\right)=0$, then

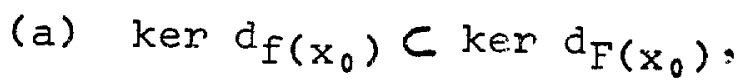

(b) there exists a Iinear function $h: G \rightarrow R$ such that $d_{F\left(x_{0}\right)}=h\left(d_{f\left(x_{0}\right)}\right)$. 
Proof: Let $S=\{x \in A: f(x)=0\}$. Then if $q$ is a unit vector belonging to ker $d_{f\left(x_{0}\right)}$, then there is a subset of $s$ having a tangent ray at $x_{0}$ with direction $q$, since $\phi\left(x_{0}+t q\right)=0$ for all $t \in R$. Therefore, there exists $S_{0} \subset S$ such that $x_{0} \in \bar{S}_{0} \backslash S_{0}$ and such that $\left[x_{0}+t y-x_{0}\right] /\left[\left\|x_{0}+t y-x_{0}\right\|\right] \rightarrow q$ as $x+t y \rightarrow x_{0}$ in $s_{0}$. Since $d_{F\left(x_{0}\right)}$ is continuous, then by the same axgument as used in Theorem 2.9

$x_{x_{0}+t y \rightarrow S_{0}} \lim _{x_{0}+t y \in S_{0}} \frac{F(x+t y)-F(x)}{\left\|x_{0}+t y-x\right\|}=d_{F\left(x_{0}\right)}(q)$. AIso since $-q \in$ ker $d_{f\left(x_{0}\right)}$, there exists $B \subset s$ such that $x_{0} \in \bar{B} \backslash B$ and that $\lim _{\substack{x_{0}+t y \rightarrow x_{0} \\ x_{0}+t y \in B}} \frac{F\left(x_{0}+t y\right)-F\left(x_{0}\right)}{\left\|x_{0}+t y-x_{0}\right\|}=d_{F\left(x_{0}\right)}(-q)=-d_{F\left(x_{0}\right)(q) .}$

But if $\mathrm{F}$ has a conditional extreme, then the above Iimits are either both non-positive or both non-negative and, therefore, $d_{F(x)} q=0$, which implies ker $d_{f(x)} \subset$ ker $d_{F(x)}$. To show part $(b)$ of this theorem, consider $h=\left\{\left(d_{f\left(x_{0}\right)}(y), d_{F\left(x_{0}\right)}(y)\right): y \in E.\right\}$ Since $d_{f\left(x_{0}\right)}$ is onto $G$, then $h$ is defined on $G$. Also $h$ is single valued since if $f e \in G, e=a_{f\left(x_{0}\right)}\left(y_{1}\right)$ and $e=d_{f\left(x_{0}\right)}\left(y_{2}\right)$,

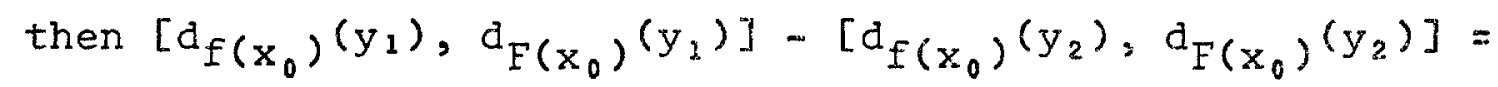

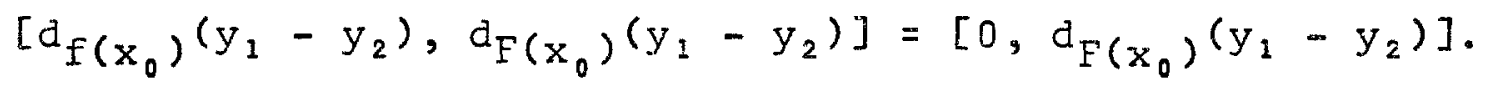
Also since $y_{1}-y_{2} \in \operatorname{ker} d_{f\left(x_{0}\right)}$, then $y_{1}-y_{2} \in \operatorname{ker} d_{F\left(x_{0}\right)}$; so $d_{F\left(x_{0}\right)}\left(y_{2}-y_{2}\right)=0$. The linearity of $h$ follows from the fact that $d_{f\left(x_{0}\right)}$ and $d_{F\left(x_{0}\right)}$ are linear. 
Theorem 2.12: Let $C$ be a closed subset of $E$ and Iet $f: \quad c \rightarrow c$ such that $\|f(x) \cdots f(y)\| \leq k\|x-y\|$ where $0 \leq k<1$. Then there exists one and only one $x \in C$ such that $f(x)=x$.

Proof: There can be onjy one such $x$, for if $f(x)=x$ and $f(y)=y$, then

$$
\|\mathrm{x}-\mathrm{y}\|=\|f(\mathrm{x})-f(\mathrm{y})\|<\mathrm{k}\|\mathrm{x}-\mathrm{y}\|<\|\mathrm{x}-\mathrm{y}\| \text {. }
$$

Let $a$ be any element of $c$, and consider the following sequence: $x_{1}=f(a), . ., x_{n}=f\left(x_{n-1}\right), .$. Clearly, if $\left\{x_{n}\right\}_{n=1}^{\infty} \rightarrow x$, then $\left\{f_{\left(x_{n}\right)}\right\}_{n=1}^{\infty}+x$.

$$
\begin{aligned}
& \left\|x_{1}-x_{2} \mid=\right\| f(a)-f\left(x_{1}\right)\|\leq k\| a-x_{1} \| \\
& \left\|x_{2}-x_{3}\right\|=\left\|f\left(x_{1}\right)-f\left(x_{2}\right)\right\| \leq k\left\|x_{1}-x_{2}\right\| \leq k^{2}\left\|a-x_{1}\right\|
\end{aligned}
$$$$
\left\|x_{n}-x_{n-1}\right\|=\left\|f_{\left(x_{n-1}\right)}-f_{\left(x_{I}\right)}\right\| \leq k\left\|x_{n-1}-x_{n}\right\| \leq k^{n}\left\|a-x_{1}\right\|
$$

If $n>m$, then $\left\|x_{m}-x_{n}\right\| \leq \sum_{j=m}^{n-1}\left\|x_{j}-x_{j+I}\right\| \leq$

${ }_{j=m}^{n-I_{k} j}\left\|a-x_{1}\right\|$. Since $\sum_{j=1}^{\infty} k^{j}$ converges for $0 \leq k<1$, then for large $m, \sum_{j=m}^{\infty} k^{j}<\varepsilon /\left\|a-x_{l}\right\|$, and this implies $\sum_{j=m}^{\infty} k^{j}\left\|a-x_{1}\right\|<\varepsilon$ which implies $\left\|x_{m}-x_{n}\right\|<\varepsilon$, so 
$\left\{x_{n}\right\}_{n=i}^{\infty}$ is a Cauchy sequence, and hence has a limi.t, $x_{0}$, and $\left\{f\left(x_{n}\right)\right\}_{n=1}^{\infty}+x_{0}$; therefore, $f\left(x_{0}\right)=x_{0}$.

Theorem 2.13: Let $g: E \rightarrow E$, and suppose for scme $a \in A, d_{g(a)}(y)=y$ for all $y \in E$. Suppose also for every $\varepsilon>0$, there exists $\delta>0$ such that if $\| x+$ ty $-a \|<\delta$ and $\|x-a\|<\delta$, then $\left\|g(x+t y)-g(x) t d_{g(a)}(y)\right\| \leq \varepsilon\|t y\|$. Then $g$ has a local inverse defined on a neighborhood of $b=g(a)$.

Proof: Let $0<\varepsilon<1$; then there exists $\delta>0$ such that if $x+t y, x \in B(a, \delta)$, then $-\varepsilon\|t y\| \leq\left\|g(x+t y)-g(x)-t d_{g(a)}(y)\right\| \leq \varepsilon\|t y\|$. By hypothesis, $\mathrm{d}_{g(a)}(y)=y$; therefore, the above becomes $-\varepsilon\|t y\| \leq\|g(x+t y)-g(x)-t y\| \leq \varepsilon\|t y\|$, and $(I-\varepsilon)\|t y\| \leq\|g(x+t y)-g(x)\| \leq(I+\varepsilon)\|t y\|$. From the inequality $(I-\varepsilon)\|t y\|<\|g(x+t y)-g(x)\|$, $g$ must be one to one on $B(a, \delta)$, for if $t_{0} \neq z$ and $g(z)=g\left(t_{0}\right)$ where $z, t_{0} \in B(a, \delta)$, then $0=\left\|g(z)-g\left(t_{0}\right)\right\|=$ $\left\|g\left(t_{0}+I\left(z-t_{0}\right)\right)-g\left(t_{0}\right)\right\| \geq(I-\varepsilon)\left\|z-t_{0}\right\|>0$. Letting $x=a$ and using $\|g(x+t y)-g(x)\| \leq(I+\varepsilon)\|t y\|$, it is found that $\|g(a+t y)-g(a)\| \leq(1+\varepsilon)\|t y\|$, and since $a+t y \in B(a, \delta)$ which implies if $\|$ ty $\|<\delta$, then $\|g(a+t y)-g(a)\| \leq$ $(1+\varepsilon) \delta$. Then $g(B(a, \delta)) \subset B(g(a),(1+\varepsilon) \delta)$.

To show $g$ is onto some neighborhood of $g(a)$, the Theorem 2.12 is used. Let $0<\delta_{1}<\delta$ and $z \in B\left(g(a),(1-\varepsilon) \delta_{1}\right)$; a.lso 
Let $F(x)=-g(x)+x+z$. Let $x_{1}=\bar{B}\left(a, \delta_{1}\right)$ and $y=B\left(a, \delta_{2}\right)$. $\mathrm{x}$ is closed, so to show the applicability of theorem 2.12 , it: must be shown that $F: x+x$. Let $x \in X$; then

$$
\begin{aligned}
& \left\|F\left(x_{0}\right)-(a)\right\|=\left\|-g\left(x_{0}\right)+x_{0}+z-a\right\|= \\
& \left\|-g\left(x_{0}\right)+g(a)+x_{0}-a+z-g(a)\right\| \leq \\
& \left\|g\left(x_{0}+I\left(a-x_{0}\right)\right)-g\left(x_{0}\right)-\left(a-x_{0}\right)\right\|+\|z-g(a)\| \leq \\
& \varepsilon\left\|a-x_{0}\right\|+(I-\varepsilon) \delta_{2} \leq \varepsilon \delta_{1}+(I-\varepsilon) \delta_{1}=\delta_{1} .
\end{aligned}
$$

The strict inequali.ty shows $F: X+X_{1}$. Also if

$s, s+\operatorname{tr} \in X$, then $s, s+\operatorname{tr} \in B(a, \delta)$. Consequently, $\|F(s)-F(s+t r)\|=\|-g(s)+s+z+g(s+t r)-(s+t r)-$ $z\|=\| g(s+t r)-g(s)-\operatorname{tr}\|\leq \varepsilon\| \operatorname{tr} \|$ for $0<\varepsilon<1$.

Thus $F$ satisfies the conditions for Theorem 2.12 , and therefore $F(x)=x$ for some $x \in X$. Since $F(x)=x$,

$-g(x)+x+z=x$, and hence $g(x)=z$ where

$z \in B\left(g(a),(I-\varepsilon) \delta_{1}\right)$. Therefore, $g$ is onto $B\left(g(a),(I-\varepsilon) \varepsilon_{1}\right)$, and each $z \in B\left(g(a),(I-\varepsilon) \delta_{1}\right)$ is some $g\left(x_{0}\right)$ where $x_{0} \in X \subset B(a, \delta)$, and $g$ is one to one on $B(a, \delta)$. Hence, $\mathrm{g}$ has a local inverse at $\mathrm{g}(\mathrm{a})$.

Theorem 2.14: Let $f: A \rightarrow F$. Let $f$ be differentiable at $a \in A$, and let $\mathrm{d}_{f(a)}^{-1}$ exist and be a bounded linear transformation from $F$ into $E$, and let $f$ satisfy the condition that for every $\varepsilon>0$, there exists $\delta>0$ such that if $\|x+t y-a\|<\delta$ and $\|x-a\|<\delta$, then

$\left\|f(x+t y)-f(x)-t d_{f(a)}(y)\right\| \leq \varepsilon\|t y\|$.

Then $f$ has a local inverse defined on a neighborhood of $f(a)$. 
Proof: Let $g=d_{f(a)}^{-1}(f)$. It will now be shown that $g$ satisfies the hypothesis of the preceding theorem. Clearly $g: E \rightarrow E$. Also $\mathrm{d}_{\mathrm{g}(\mathrm{a})}(\mathrm{y})=y$ since $d_{g(a)}(y)=\lim _{t \rightarrow 0} \frac{d_{f}^{-1}(a)(f(a+t y))-d_{f(a)}^{-1}(f(a))}{t}=$ $d_{f(a)}^{-1}\left(d_{f(a)}(y)\right)=y$. Also, by (i) there exists $\delta>0$ such that if $\|x+t y-a\|<\delta$ and $\|x-a\|<\delta$, then $\left.\left\|f(x+t y)-f(x)-t d_{f(a)}(y)\right\| \leq E /\left\|d_{f(a)}^{-1}\right\|\right]\|t y\|$. Now $\left\|g(x+t y)-g(x)-t d_{g(a)}(y)\right\|=$ $\left\|d_{f(a)}^{-1}(x+t y)-d_{f(a)}^{-1} f(x)-t d_{f(a)}^{-1} d_{f(a)}(y)\right\| \leq$ $\left\|d_{f(a)}^{-1}\right\|\left\|f(x+t y)-f(x)-t d_{f(a)}(y)\right\| \leq$ $\left\|d_{\hat{f}(a)}\right\| \varepsilon /\left\|d_{f(a)}^{-1}\right\|\|t y\|=\varepsilon\|t y\|$. Therefore, g satisfies the conditions of the previous theorem and $g$ has a local inverse $h$ defined on a neighborhood of $b=g(a)$.

The function $g$ is one to one on some set $B(a, \delta), 30 \mathrm{f}$ is one to one on $B(a, \delta)$; for if $s, t \in B(a, \delta)$ such that $f(s)=f(t)$, then $g(s)=d_{f(a)}^{-1} f(s)=d_{f(a)}^{-1} f(t)=g(t)$. Therefore, $t=s$, and hence $f$ is one to one on $B(a, \delta)$. Since $d_{f(a)}^{-1}$ is bounded, there exists $T \in R$ such that $\left\|d_{f(a)}^{-1}(w)\right\| \leq T\|w\|$ for ail $w \in F$. There exists $\delta_{1}$ such that $g$ is onto $B\left(g(a), \delta_{1}\right)$. Let $\delta_{2}=\delta_{1} / T$, and let $k \in B\left(f(a), \delta_{2}\right)$. Then $\left\|d_{f(a)}^{-1}[k-f(a)]\right\| \leq T\|k-f(a)\|<T \delta_{2}=\delta_{1}$; consequently $d_{f}^{-l}(a)(k) \in B\left(g(a), \delta_{1}\right)$. Let e exist such that $\|e-f(a)\|<\delta_{2} ;$ then $\left\|d_{f(a)}^{-1}[e-f(a)]\right\| \leq$ $T\|e-f(a)\|<T \delta_{2}=\delta_{1}$ which implies $d_{f(a)}^{-1}(e) \in B\left(g(a), \delta_{1}\right)$. 
Since $g$ is onto Big(a), $\left.\hat{\delta}_{i}\right)$, there exists $s \in B(a, \delta)$ such that $g(s)=d_{f(a)}^{-1}\left(e ;\right.$ itherfone, $\sigma_{I(a)}^{-l}(f(s))=d_{f(a)}^{-1}(e)$. Consequently $f(s)=e$, and, therefore, fis onto $B\left(f(a), \delta_{2}\right)$, and since $s \in B(a, \delta)$, then $f(B(a, \delta))>B\left(f(a), \delta_{2}\right)$. Hence $f$ has an inverse or $B\left(f(a), \delta_{2}\right)$.

Definition 2.6: If $B_{1}$ and $B_{2}$ are Banach spaces and $(x, y) \in B_{2} \times B_{2}$, then $\|(x, y)\|=\|x\|+\|y\|$.

Lemma 2.6: If $\mathrm{T}_{3}: \mathrm{B}_{1} \rightarrow \mathrm{B}_{2}$ and $\mathrm{T}_{2}: \mathrm{B}_{1} \rightarrow \mathrm{B}_{2}$ are bounded Iinear transformations, then $\mathrm{T}_{1}+\mathrm{T}_{2}$ is a bounded transformation.

Proof: Since $T_{1}$ is bounded, there exists $M_{1} \in R$ such that if $x \in B_{1},\left\|T_{1}(x)\right\| \leq M_{1}\|x\|$. A.lso there exists $M_{2} \in R$ such that if $x \in B_{1}\left\|T_{2}(x)\right\| \leq M_{2}\|x\|$, then

$$
\begin{aligned}
& \left\|T_{1}(x)+T_{2}(x)\right\| \leq\left\|T_{1}(x)\right\|+\left\|T_{2}(x)\right\| \leq \\
& \left(M_{1}+M_{2}\right)\|x\| .
\end{aligned}
$$

Lemma 2.7: If $\mathrm{T}_{1}: \mathrm{B}_{1} \rightarrow \mathrm{B}_{2}$ and $\mathrm{T}_{2}: \mathrm{B}_{2} \rightarrow \mathrm{B}_{3}$ are bounded Iinear transformations, then $\mathrm{T}_{2} \cdot \mathrm{T}_{1}$ j.s bounded.

Proof: There exists $M_{1} \in R$ such that if $x \in B_{1}$, $\left\|T_{1}(x)\right\| \leq M\|x\|$. Also there exists $M_{2} \in R$ such that if $y \in B_{2}$, then $\left\|T_{2}(y)\right\| \leq M_{2}\|y\|$

$$
\begin{aligned}
& \left\|T_{2} \cdot T_{1}(x)\right\|=\left\|T_{2}\left(T_{1}(x)\right)\right\| \leq M_{2}\left\|T_{1}(x)\right\| \leq M_{2} \cdot M_{1} \cdot\|x\| \cdot \\
& \text { Lemma 2.8: Iset } F: F \times F \rightarrow G \text {, and let } F\left(x_{0}, y_{0}\right)=0 .
\end{aligned}
$$

Let $g: F \rightarrow G$ where $g(y)=F\left(x_{0}, y\right)$, and let $f: E \rightarrow G$ where $f(x)=F\left(x, y_{0}\right)$. Let $F$ be strongly differentiable at $\left(x_{0}, y_{0}\right)$ and satisfy the following: for every $\varepsilon>0$, there exists $\delta>0$ such that if 


$$
\begin{aligned}
& \|(x, y)+t(w, z)-\left(x_{0}, y_{0} y<\hat{\theta}\right. \text { and } \\
& \left\|(x, y)-\left(x_{0}, y_{0}\right)\right\|<\delta \text {, then }
\end{aligned}
$$$$
\left\|F[(x, y)+t(w, z)]-F(x, y)-t d\left(x_{0}, y_{0}\right)(w, z)\right\|<
$$

$\varepsilon\|w, z\|$. Let $d_{g\left(y_{0}\right)}^{-1}$ exist as a bounded Iinear transformation and let $d_{f\left(x_{0}\right)}$ be bounded. If $\phi: E \times F \rightarrow E_{1} \times G$ where $\phi(x, y)=(x, F(x, y))$, then $\phi$ has a local inverse at $\phi\left(x_{0}, y_{0}\right)$.

Proof: Let $(x, y) \leq B_{1} \times B_{2}$, and let $\varepsilon>0$. There exists $\delta$ such that if $\|t(x, y)\|<\delta$, then $\left\|F\left[\left(x_{0}, y_{0}\right)+t(x, y)\right]-F\left(x_{0}, y_{0}\right)-t d^{\prime}\left(x_{0}, y_{0}\right)(x, y)\right\|<$ $\varepsilon\|t(x, y)\|$. Let $\|t(x, y)\|<\delta$, and consider $\left\|\phi\left[\left(x_{0}, y_{0}\right)+t(x, y)\right]-\phi\left(x_{0}, y_{0}\right)-t\left[x, d_{F\left(x_{0}, y_{0}\right)}(x, y)\right]\right\|$ $=\|\left(0, F\left[\left(x_{0}, y_{0}\right)+t(x, y)\right]-F\left(x_{0}, y_{0}\right)-t d_{F\left(x_{0}, y_{0}\right)}(x, y) \|\right.$ $=\|0\|+\| F\left[\left(x_{0}, y_{0}\right)+t(x, y)-F\left(x_{0}, y_{0}\right)-t d_{F}\left(x_{0}, y_{0}\right)(x, y)\right.$ $\leq \varepsilon\|t(x, y)\|$. Hence $d_{\phi\left(x_{0}, y_{0}\right)}(x, y)=\left(x, d_{F\left(x_{0}, y_{0}\right)}(x, y)\right)$. Since $F$ is strongly differentiable at $\left(x_{0}, y_{0}\right)$, $\mathrm{d}_{F\left(x_{0}, y_{0}\right)}(x, y)=d_{F\left(x_{0}, y_{0}\right)}(x, 0)+d_{F\left(x_{0}, y_{0}\right)}(0, y)=$ $d_{f\left(x_{0}\right)}(x)+d_{g\left(y_{0}\right)}(y)$. Then if $(w, z) \in E \times G$, $d_{\phi}^{-1}\left(x_{0}, y_{0}\right)(w, z)=\left(w, d_{g\left(y_{0}\right)}^{-1}\left[z-d_{f\left(x_{0}\right)}(x)\right]\right)$, for by the

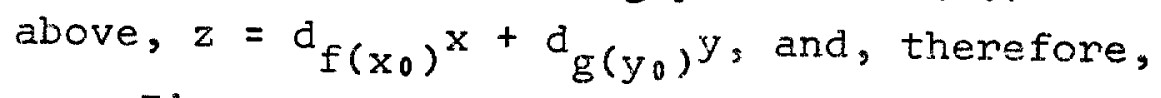
$y=d_{g\left(y_{0}\right)}^{-1}\left[z-d_{f\left(x_{0}\right)}\left(x^{\prime}\right)\right]$. Since $d_{g\left(y_{0}\right)}^{-1}$ and $d_{f\left(x_{0}\right)}$ are linear, then $d_{\phi}^{-1}\left(x_{0}, y_{0}\right)$ is Iinear.

Since $d_{f\left(x_{0}\right)}$ is bounded, then by Lemma $2.6,(w, z) \stackrel{T^{*}}{ }$ $z-d_{f\left(x_{0}\right)}(w)$ is bounded and by Lemma 2.7 , $(w, z) \stackrel{\mathrm{T}_{2}}{\rightarrow} \mathrm{d}_{\mathrm{g}\left(\mathrm{y}_{0}\right)}\left[z-\mathrm{d}_{\mathrm{f}\left(\mathrm{x}_{0}\right)}(w)\right]$ is bounded. Therefore, there 
exists $M_{3} \in R$ such that if $(w, z) \in B_{1} \rightarrow B_{3}$, then $\left\|d_{g\left(y_{0}\right)}\left[z-d_{f\left(x_{0}\right)}(w)\right]\right\| \leq M_{I}\|(w, z)\|$. Consequently, $\left\|d_{\phi\left(x_{0}, y_{0}\right)}^{(w, z)}\right\|=\|w\|+\left\|d_{g\left(y_{0}\right)}^{-1}\left[z-a_{f\left(x_{0}\right)}(w)\right]\right\| \leq$ $\|(w, z)\|+M_{1}\|(w, z)\|=\left(1+M_{1}\right)\|(w, z)\|$. Therefore, $\left.d_{\phi\left(x_{0}\right.}^{-1}, y_{0}\right)$ is bounded. Also, given $\varepsilon>0$, there exists $\delta>0$ such that if $[(x, y)+t(w, z)],[x, y] \in B\left[\left(x_{0}, y_{0}\right), \delta\right]$, then $\left\|F[(x, y)+t(w, z)]-F(x, y)-t d F\left(x_{0}, y_{0}\right)^{(w, z)}\right\| \leq$ $\varepsilon\|t(w, z)\|$. Let $[(x, y)+\tau(w, z)],[x, y] \in B\left[\left(x_{0}, y_{0}\right), \delta\right]$. Then $\left\|\phi[(x, y)+t(w, z)]-\phi(x, y)-t d_{\phi\left(x_{0}, y_{0}\right)}(w, z)\right\|=$ $\left\|\left(0, F(x, y)+t(w, z)-F(x, y)-t d_{\phi\left(x_{0}, y_{0}\right)}(w, z)\right)\right\| \leq$ $\varepsilon\|t(w, z)\|$. Since $\phi$ satisfies the hypothesis of Theorem 2.14 , $\phi$ has a local inverse at $\phi\left(x_{0}, y_{0}\right)$.

Theorem 2.15: Let $F, g$, and $f$ satisfy the hypothesis of Lemma 2.8; then there exists $\delta_{1}, \delta_{2}>0$, such that if $\left\|x-x_{0}\right\|<\delta_{2}$, there exists one and only one $y$ such that $\left\|y-y_{0}\right\|<\delta_{1}$ and $F(x, y)=0$.

Proof: Let $\phi$ be as in Lemma 2.8. Then there exists $\delta_{1}, \delta_{2}>0$ such that $B\left[\left(x_{0}, y_{0}\right), \delta_{1}\right] \Phi\left(B_{1} \rightarrow B_{3}\right)$ and $\phi$ is one to one on $B\left[\left(x_{0}, y_{0}\right), \delta_{1}\right]$ and onto $B\left[\phi\left(x_{0}, y_{0}\right), \delta_{1}\right]$. $\phi\left(x_{0}, y_{0}\right)=\left[x_{0}, F\left(x_{0}, y_{0}\right)\right]=\left(x_{0}, 0\right)$; therefore if $\left\|x-x_{0}\right\|<\delta_{2}$, then $\left\|(x, 0)-\left(x_{0}, 0\right)\right\|<\delta_{2}$; consequently there exists $(x, y)$ such that $(x, y)=\phi^{-1}(x, 0)$ and $(x, y) \quad B\left[\left(x_{0}, y_{0}\right), \delta_{1}\right]$. Hence, $\left\|y-y_{0}\right\|<\delta_{1}$. Since $\phi(x, y)=(x, 0), F(x, y)=0$. 


\section{BIDLIOGRAPHY}

Brown, A. L. and A. Page, Elements of Functional Arialysis, London, Van Nostrand Reinholt Company, 1970.

Flett, T. M., "On Differentiation in Nomed Vector Spaces," Journal London Mathematical Society, XLII $(1967), 523$.

Smith, Kennant, Primer of Modern Analysis, Bogden \& Quigley, Belmont, Californía, I97I. 